\title{
The Use of a Specialized Nutritional Supplement for Diabetic Foot Ulcers Reduces the Use of Antibiotics
}

\author{
Patrizio Tatti ${ }^{\mathrm{a}, \mathrm{c}}$, Annabel Elisabeth Barber ${ }^{\mathrm{b}}$
}

\begin{abstract}
Background: Diabetic foot ulcers (DFU) are among the most invalidating and costly complications of diabetes. We evaluated the effect of a blend of arginine, glutamine and $\beta$-hydroxy- $\beta$ methylbutyrate on the healing of these lesions in a group of 22 diabetic subjects who served as their own control. A nutritional supplement containing arginine, glutamine and beta-hydroxy-beta-methylbutyrate (Ar-Gl-HMB) dramatically improved the healing of diabetic foot ulcers in two studies. Glutamine and Arginine have an antibacterial and immunopotentiating action and should reduce the use of antibiotics. We evaluated the use and the cost of antibiotics using the data from two case controlled studies of diabetic subjects with recurrent neuropathic diabetic foot ulcers.
\end{abstract}

Methods: We identified 22 subjects with type- 2 diabetes who had been treated in the past for neuropathic ulcers of the foot (Event 1, E1), and who subsequently suffered recurrent ulcers with similar characteristics (E2). In E2 the treatment was the same but the subjects received a supplementation of Ar-Gl-HMB, and experienced $50 \%$ reduction of time to healing.

Results: During E1 we used 83 courses of antibiotics, versus 36 during E2 (Wilcoxon SR test $=0.002$ ). The cost for treatment E1 was $17123.77 €$, versus $8537.8 €$ in E2. The difference persists also

\footnotetext{
Manuscript accepted for publication February 3, 2012

${ }^{a}$ Department of Surgery, University of Nevada School of Medicine, Las Vegas, USA

${ }^{\mathrm{b}}$ Department of Diabetes and Endocrinology, ASL RMH, Roma, Italy

${ }^{\mathrm{c} C}$ Corresponding author: Patrizio Tatti. Email: info@patriziotatti.it
}

doi:10.4021/jem64w adding the cost of the supplement of $2586 €(8537.8+171223.77$ $=1123.8 €)$.

Conclusions: The cost of the antibiotic treatment was reduced by $50 \%$ with the use of the formula. Because the time to healing was shortened, both the direct costs (medication, surgery, nursing time, etc) and the indirect costs (hospitalization, days of work lost) were decreased .The use of a highly specialized nutrition reduces the cost of the antibiotic treatment by about $50 \%$ and the auxiliary economic and social burden of disease.

Keywords: Diabetic foot ulcer; Infection; Antibiotics

\section{Introduction}

Diabetic foot ulcers (DFU) are among the most invalidating and costly complications of diabetes [1]. These ulcers appear in the diabetic environment and should be kept separated from the ulcers appearing in non diabetics. Since this environment is the sine qua non for the appearance of these lesions, we suggest that the term "treatment of the DFU" is a misnomer and should be substituted with "treatment of the diabetic subject with a foot ulcer" [2]. These ulcers are of two main types, of vascular and neuropathic origin, and in many cases, there may be an overlap of these conditions [3]. The initiating cause of the ulcers may be any event that occurs also in nondiabetics, like trauma, pressure or burn. Independent of their origin these ulcers share the common characteristic of a delayed healing in comparison with the same lesions appearing in nondiabetic individuals.

DFUs have a high social and personal cost [4]. There are limited data in the literature on the topic of the economic cost. Furthermore, the biology and the nutritional status of the patient may interfere with the healing process. Because of this complexity any economic evaluation is a generalization. A retrospective study of men $40-64$ years of age in a managed care population found that the attributable cost for foot ulcer care was $\$ 27,987$ [5]. According to another study, the total direct costs for healing of infected ulcers not requiring amputation were 17500 \$; the costs for lower extremity 


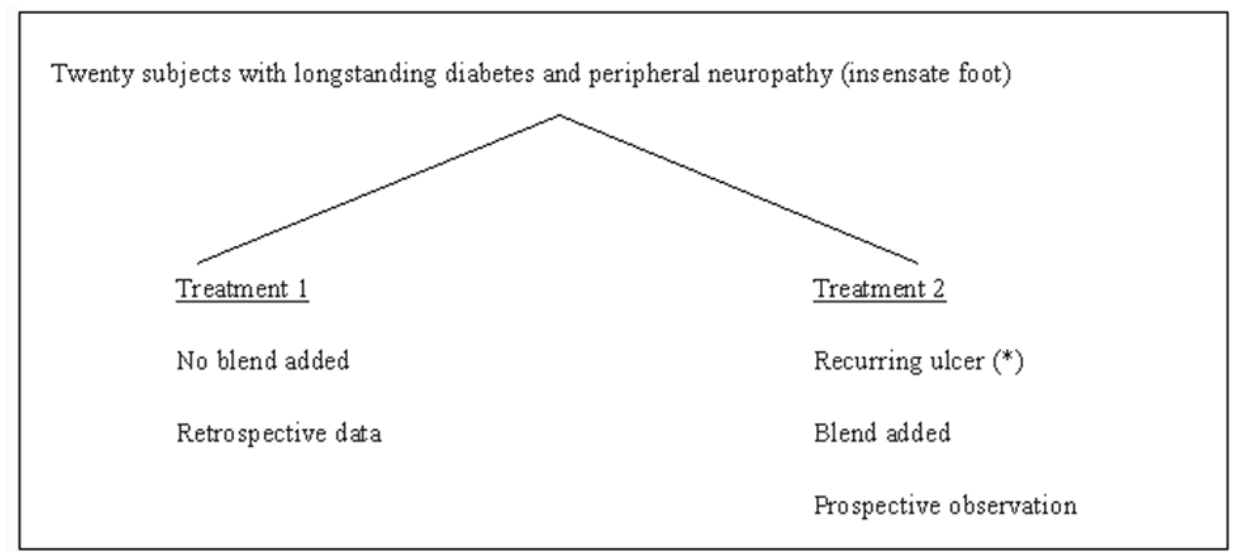

Figure 1. Description of the study.

amputation were $30000-35000 \$[6]$.

The present paper focuses on the neuropathic DFU in a population of longstanding diabetic patients in an age range of $45-70$ years. The data of 12 subjects included for this economic analysis were described in a 2010 paper [7], other 10 were presented in 2011 at the EWMA meeting in Brussels [8].

\section{Aim \\ We decided to evaluate the cost-benefit ratio of a blend of arginine, glutamine and HMB used to improve the healing of diabetic foot ulcers. In a previous study we demonstrated that this blend could reduce the time to healing in diabetic subjects with neuropathic foot ulcers from 247 days to 83 days [7].}

\section{Summary of previous studies}

We analyzed the data on the outcome of 22 longstanding diabetic subjects with peripheral neuropathy and a relapsing diabetic foot ulcer from two previous studies [7, 8]. These subjects suffered in the past a DFU treated conventionally
(Event 1, Fig. 1) with curettage, local antisepsis and antibiotics. After the healing, either spontaneous or following a surgical procedure, the ulcer recurred in the same location and was treated with an oral blend of Arginine, glutamine and $\beta$-hydroxy- $\beta$-methylglutarate added on top of the same care used for the previous Event (Event 2). The data of the two events (E1 and E2) were compared. We considered these subjects eligible for this study if the size of the recurring ulcer was the same $\pm 1 \mathrm{~mm}$. Each ulcer was accurately photographed at the different stages of evolution. The choice of this protocol was based on some considerations: (1) this design is reflective of what happens in clinical practice. (2) the use of the same subject with a recurring lesion with identical characteristics allows a perfect biologic match for a condition with an enormous number of variables.

All these subjects were treated with one or more of three different antibiotics according to the clinical judgment and a protocol included in the ISO manual of Accreditation, Ed 3.1 for internal use. In all these patients the time to healing and the use of antibiotics was accurately recorded.

The male to female ratio was $7 / 15$, the age range was $45-71$, mean $=64$. Fifteen of these subjects were retired, the others actively working in commerce or manual work.

Table 1. Main Results

\begin{tabular}{lll}
\hline & First ulcer & Recurring ulcer \\
\hline Age (ys) & $64.8 \pm 11$ & $65.2 \pm 7$ \\
$\begin{array}{l}\text { HbAlc (mmol/mol) at the time of presentation of } \\
\text { the ulcer }\end{array}$ & $77.04 \pm 15$ & $78.1 \pm 0.18(\mathrm{P}=\mathrm{NS})$ \\
$\begin{array}{l}\text { Duration of the ulcer } \\
\text { BCMI }^{\mathrm{a}}\end{array}$ & $215 \pm 48$ & $81 \pm 16(\mathrm{P} \leq 0.01)$ \\
\end{tabular}

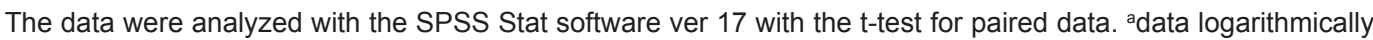
transformed for calculation. 
Table 2. Antibiotics Used and the Relative Cost per Course

\begin{tabular}{lll}
\hline & Without Ar-G-B & With Ar-G-B \\
\hline Total number of cycles of antibiotics & 83 & 36 \\
Total number of cycles of antibiotic A & $34(3042 €)$ & $20(1789.6 €)$ \\
Total number of cycles of antibiotic B & $34(4719.2 €)$ & $12(1665.6 €)$ \\
Total number of cycles of antibiotic C & $15(9362.25 €)$ & $4(2496.6 €)$ \\
Total cost of antibiotics & $17123.77 €$ & $5951.8 €$ \\
Cost of the nutritional supplement & 0 & $2586 €$ \\
Total cost of treatment (antibiotics + supplement) & $17123.77 €$ & $8537.8 €$ \\
\hline
\end{tabular}

Legend: Cost per cycle of the three antibiotics used: $A=89.48 € ; B=138.8 € ; C=624.15 €$.

\section{Materials and Methods}

Before each period of treatment all patients underwent a general physical examination and a routine check for blood glucose,HbAlc, serum creatinine, complete blood count, protein electrophoresis, electrolytes, and overnight (12 h) urinary protein excretion. They also monitored their blood glucose levels regularly. At the first visit, the ulcer was photographed and measured. Body composition was also measured using bioelectrical impedance analysis (BIA) with the Akern Analyzer BIA 101 using the method described by Dittmar. Among the BIA parameters we weekly monitored the Body Cell Mass Index (BCMI). The medications were adjusted to improve diabetes management and hypertension as needed. Curettage was performed if deemed appropriate by the physician. Wounds were cultured and infected wounds were treated with appropriate antibiotics. Follow up visits were scheduled at weekly intervals. At each visit the treatment was further adjusted if indicated. In addition the patients were referred to the dietician who determined their energy needs based on age and activity level. For the overweight or obese patients, a slightly hypo-caloric diet, with $20 \%$ reduction of total intake was prescribed, and a highly nutritional formula was added. The recommended protein content of the diet was at least $20 \%$ taken at the three daily meals. The dietary prescriptions were the same as for the previous ulcer event (treatment 1, Fig. 1). The patients were followed up weekly until their wounds were closed. For the treatment of recurring DFU (Treament 2), the formula was started at the second visit. Thus the only difference between the treatment periods (treatment 1 vs 2 ) was the addition of $\mathrm{HMB}$, glutamine and arginine supplementation during treatment 2. All the clinical data were recorded in the patients' electronic records together with the laboratory values and the results of the body composition analysis. The time when the patients first observed the presence of wounds was used to calculate the time of the first medical evidence of the disease. The details of these studies have already been presented.

Since the protocols used for both studies were identical we created a single database of 22 subjects. The data on the antibiotics used were obtained from the electronic medical records and cross checked with the pharmacy records. The data reported in Table 1 were analyzed with the T test for paired samples (SPSS 19 package). The data on the use of antibiotics that are pertinent to this study are in Table 2 . The data were analyzed per valuable cohort (per protocol), not ITT since the compliance was $100 \%$. The compliance was regularly evaluated since $90 \%$ of the treatments were in day hospital setting, and for the remaining $10 \%$ the subjects in treatment retuned the empty boxes/vials of antibiotics.

\section{Use of antibiotics}

In our institution whenever needed we use three antibiotics in courses according to what is stated in the ISO protocol ver 3.1 for internal use which was the same during treatment 1 and 2 .

\section{Results}

The main results reported in table 1 are the reduction in time to healing from 215 to 81 days $(1 / 3), P=0.001$, and the increased Body Cell Mass Index (BCMI). The BCMI is a measure derived from the BIA and is the normalization of the Body cell mass for the height of the subject. The number of cycles of antibiotics and the cost of the treatments were reduced to nearly one third (Table 2). In monetary terms 


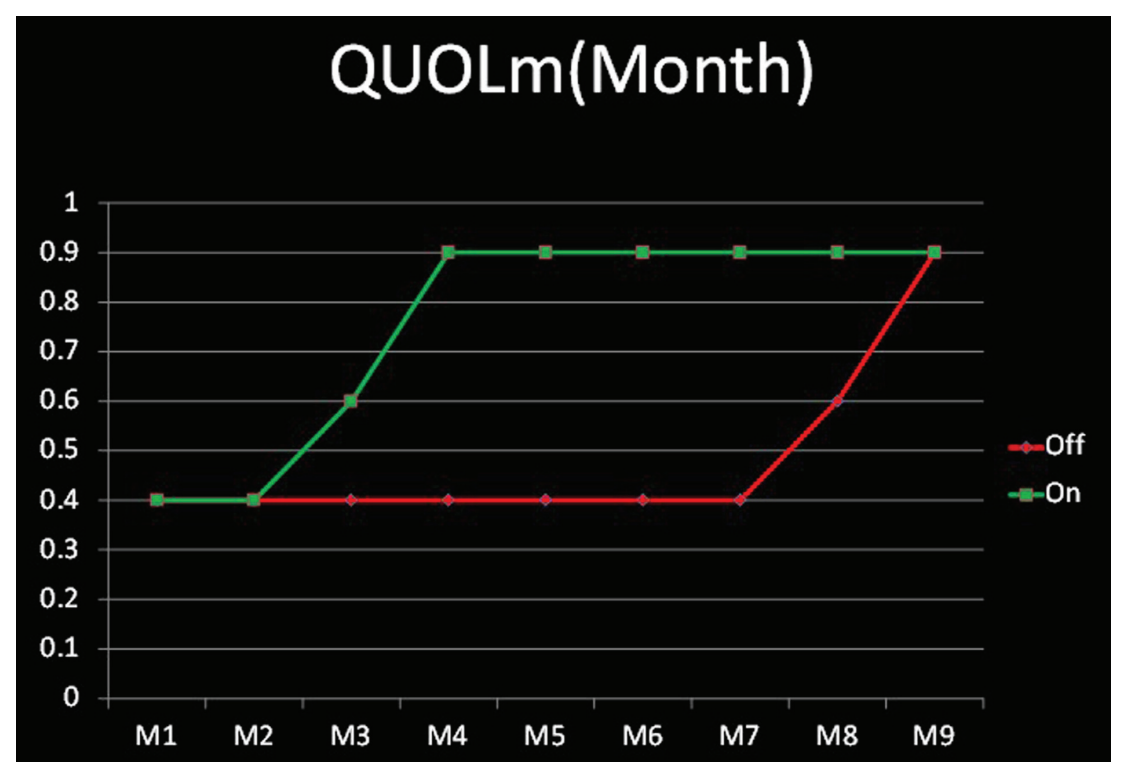

Figure 2. Quality of life/month.

$8537.8 €$ vs. $17123.77 €$. Throughout the treatment 2 there was also shift towards the less potent and less expensive drugs. As reported in table 2 the number of cycles of antibiotic 1 , with the minor cost was only slightly reduced from 34 to 20 , while the cycles of the other two were reduced to less than $1 / 3$.

The compliance of the patient was evaluated by the nurses in the hospital setting and by the empty boxes returned in the outpatient setting. The need for antibiotics was evaluated by the same two physicians who treated the previous infection and according to the laboratory results of the antibiotic sensitivity tests Table 2 is a summary of the actual use of antibiotics. To these should be added the direct costs (medication, surgical interventions and dressings, nursing time, etc) and the indirect costs (hospitalization, days of work lost for the patient and the relatives). The reduction in antibiotic usage can be only partially explained by the reduction in time to healing. During the treatment period there was a shift towards the antibiotic A, of lower cost and minor spectrum and power, since the infectious process was less aggressive. This is entirely consistent with the antibacterial and immunogenic properties of the blend we used and with the improved nutrition.

\section{Quality of life}

To evaluate the change in quality of life we asked the patients and their family members to fill a series of EQ-5D questionnaires (http://www.euroqol.org/fileadmin/user_upload/ Documenten/PDF/Languages/Sample_UK_English_EQ5D-3L.pdf) in the Day Hospital setting. The questionnaires were auto administered with the help of a nurse only when required. According to the results we considered the pres- ence of an ulcer of the foot a reduction in quality of life of 0.4 . Due to the wide variety of ulcers and the role of social/ working context this figure is obviously an average. Since the healing process started after the first month in the treated group (E2) we estimated an initial increase to 0.6 and a final increase to 0.9 in the second month. Although there was complete healing the need for a continuous effort to prevent the recurrences fixed this improvement to 0.9 rather than 1 (Fig. 2). The delayed and incomplete healing in the off treatment period explains the final QUOL $=0.7$. Since the period of observation is calculated in months and not in years we used the term QUOLm, where $\mathrm{m}=$ month.

We also calculated the cost: effectiveness ratio of the treatment with the formula. The formula was qualy $\times$ months of extra time to healing of treatment $1 \mathrm{vs}$ treatment 2 . Treatment 1: $0.4 \times 4=1.6$; Treatment $2: 0.9 \times 4=3.2$.

In other words treatment 2 allowed a gain of 4 extra months at a quasi normal state

Since the average cost per patient in treatment 1 is $1426.917 €(1723.77) / 12$ and for treatment 2 is 711.4833 $(8537.8 / 12)$ the net difference is a saving of $178 €$ per month for the antibiotic treatment while obtaining a 0.9 QOL. The extra costs for the medications of a nonhealing ulcers and the time lost are not included in this calculation.

\section{Discussion}

During the period of treatment of the recurring ulcers (treatment 2) with the addiction of a blend of Arginine, Glurtamine and $\mathrm{HMB}$, we used nearly $1 / 3$ of the antibiotics used in absence of the blend (treatment 1). Notably in treatment 2 we shifted towards the oral treatment and the less costly and less 


\section{Determinants of cost $\quad$ Antagonist effect}

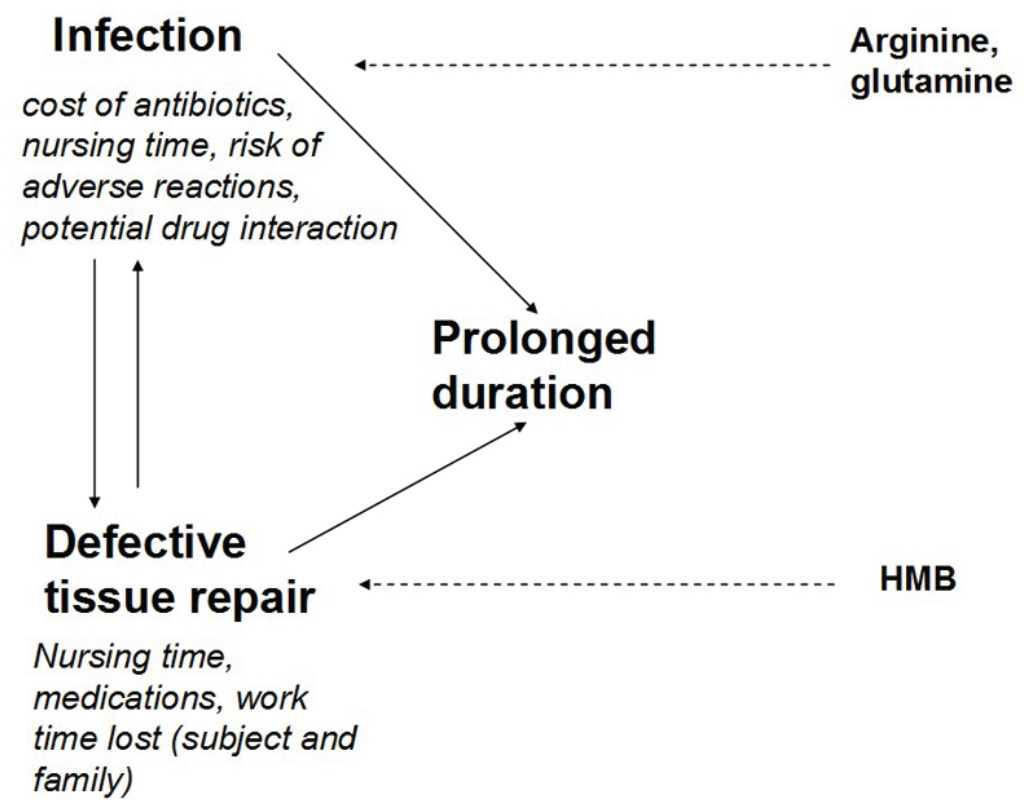

Figure 3. Economic impact of the ulcer.

powerful antibiotics. Such difference probably reflects a reduced aggressiveness of the infection, and a more powerful immune host response. This also led to a consistent reduction in costs of the antibiotics and all the accessory therapy (nursing, requirements for the injections) [9]. Of course the rapid healing of the ulcer during treatment 2 allowed for a considerable saving in costs of local medications and the hospital personnel, and time lost both for the patient and his family, and should be added to the economic advantage. In summary the addiction of the blend reduced the economic and social costs of the ulcer both shortening the time to healing and allowing for the use of less, and less costly antibiotics. This result is probably due to the interaction of many factors (Fig. $3)$. We think that argine in the blend reduced the bacterial load and potentiated the immunologic process [10-12], thus reversing the infection/inflammation and allowing the repair of the tissue. Arginine also has a vasodilator action [13] that was exploited for male erectile dysfunction before the introduction of the phosphodiesterase inhibitors. Glutamine also has a spectrum of positive effects [14], notably stimulation of protein synthesis, support of the immune function [15, 16] and of the intestinal mucosal integrity [17]. HMB is normally present in the muscle, is an active leucine metabolite and a potent stimulator of mTOR (Mammalian Target of Rapamycin), an intracellular protein controlling protein synthesis $[18,19]$. Due to its anabolic property, the substance is widely used by the athletes worldwide and to reverse the effects of aging [20]. In our experience the use of this blend reduced to $1 / 3$ the time to healing in a group of 12 diabetic patients while improving the lean body mass [21].

This study has both weaknesses and strengths. This is not a Randomized Controlled Study (RCS). However the RCTs that are considered the gold standard have enormous difficulties with ulcers appearing in diabetic subjects, because the evolution of the ulcers is conditioned by a variety of factors that are extremely difficult to keep under control [22]. Among them the intrinsic variability of the wound, the presence of infections, the location of the wound, the use of ill fitting shoes, time spent with the shoes, the general medical condition of the subjects and the blood glucose control. This diversity can be partially reduced by the use of one of the many classifications available in the literature, but no one of them accounts for the wide spectrum of concomitant conditions that can interfere with the healing process. One of the most important and often overlooked among these factors is the glycemic control. HbA1c is considered the gold standard in evaluating glycaemic control in a diabetic patient, but may be variably influenced by both fasting and postprandial blood glucose levels, and each of these has a different pathophysiological meaning. Currently we do not even know if and to what extent these phenomena can interfere with the healing of the ulcer. It is also probable that we ignore some of the individual genetic or acquired characteristics that can interfere with the tissue repair. Thus is extremely difficult to find a population with the same characteristics and an effective randomization would require a very large number of subjects. Consequently our choice of subjects affected by a recurring ulcer with the same characteristics allows to keep 
under control most of the biologic variability of the ulcer.

The incidence of DFUs is very high. In a recent study, among 8,905 patients identified with type 1 or type 2 diabetes, 514 developed a foot ulcer over 3 years of observation (cumulative incidence 5.8\%) [22], and a reduction of nearly $50 \%$ in the cost has an important bearing of the national health system. The use of this blend may help reduce these costs while improving the well being of the diabetic subjects.

\section{References}

1. Ortegon MM, Redekop WK, Niessen LW. Cost-effectiveness of prevention and treatment of the diabetic foot: a Markov analysis. Diabetes Care. 2004;27(4):901-907.

2. Tatti P, Barber A, Role of nutrition in ulcer healing. In "Diabetic Foot". ISBN 978-953-307-717-8.

3. American Diabetes Association. Clinical Care of the diabetic Foot, 2nd Edition (2010).

4. Holzer SE, Camerota A, Martens L, Cuerdon T, CrystalPeters J, Zagari M. Costs and duration of care for lower extremity ulcers in patients with diabetes. Clin Ther. 1998;20(1):169-181.

5. Ramsey SD, Newton K, Blough D, McCulloch DK, Sandhu N, Reiber GE, Wagner EH. Incidence, outcomes, and cost of foot ulcers in patients with diabetes. Diabetes Care. 1999;22(3):382-387.

6. Ragnarson Tennvall G, Apelqvist J. Health-economic consequences of diabetic foot lesions. Clin Infect Dis. 2004;39 Suppl 2:S132-139.

7. Tatti P, Barber A. The use of a specialized nutritional supplement for diabetic foot ulcers reduces the use of antibiotics and the cost of treatment. Presented at the EWMA 2011, 25-27 May, Brussels.

8. Tatti P, Barber A. EWMA Journal, Nutritional Supplement is Associated with a Reduction in Healing Time and Improvement of Fat Free Body Mass in Patients with Diabetic Foot Ulcers. Vol 10(3), Oct 2010:13-18.

9. Standards of medical care in diabetes--2011. Diabetes Care. 2011;34 Suppl 1:S11-61.

10. Wanasen N, Soong L. L-arginine metabolism and its impact on host immunity against Leishmania infection. Immunol Res. 2008;41(1):15-25.

11. Barbul A, Lazarou SA, Efron DT, Wasserkrug HL, Efron
G. Arginine enhances wound healing and lymphocyte immune responses in humans. Surgery. 1990;108(2):331336; discussion 336-337.

12. Witte MB, Barbul A. Arginine physiology and its implication for wound healing. Wound Repair Regen. 2003;11(6):419-423.

13. Rhodes P, Barr CS, Struthers AD. Arginine, lysine and ornithine as vasodilators in the forearm of man. Eur $\mathrm{J}$ Clin Invest. 1996;26(4):325-331.

14. Peng X, Yan H, You Z, Wang P, Wang S. Clinical and protein metabolic efficacy of glutamine granules-supplemented enteral nutrition in severely burned patients. Burns. 2005;31(3):342-346.

15. Wilmore DW. The effect of glutamine supplementation in patients following elective surgery and accidental injury. J Nutr. 2001;131(9 Suppl):2543S-2549S; discussion 2550S-2541S.

16. Mocchegiani E, Nistico G, Santarelli L, Fabris N. Effect of L-arginine on thymic function. Possible role of L-arginine: Nitric oxide (no) pathway. Arch Gerontol Geriatr. 1994;19 Suppl 1:163-170.

17. van der Hulst RR, van Kreel BK, von Meyenfeldt MF, Brummer RJ, Arends JW, Deutz NE, Soeters PB. Glutamine and the preservation of gut integrity. Lancet. 1993;341(8857):1363-1365.

18. Manzano $M$, et $A 1$. Is $\beta$-hydroxy- $\beta$-methylbutyrate (HMB) the bioactive metabolite of L-leucine (LEU) in muscle? Molecular evidence and potential implications. Abstract presented at: European Society for Clinical Nutrition and Metabolism 31st Congress; Vienna, Austria; August 29-September 1, (2009). Abstract P267.

19. Baxter J et Al. 4th cachexia conference 2007, Abstract 1.18 .

20. Wilson GJ, Wilson JM, Manninen AH. Effects of betahydroxy-beta-methylbutyrate (HMB) on exercise performance and body composition across varying levels of age, sex, and training experience: A review. Nutr Metab (Lond). 2008;5:1.

21. Tatti P, Barber AB. Nutritional supplement is associated with a reduction in healing time and improvement of fat-free body mass in patients with diabetic foot ulcers. EWMA Journal vol 10 no 3(2010): 13-17.

22. Armstrong DG, Lavery LA. Diabetic foot ulcers: prevention, diagnosis and classification. Am Fam Physician. 1998;57(6):1325-1332, 1337-1328. 